\title{
Atypical ameloblastoma - an enigma in diagnosis: review of literature and report of a case
}

\author{
Thomas George ${ }^{1}$, Kundendu A. Bishen ${ }^{2}$ \\ ${ }^{1}$ MDS, Visiting senior lecturer, Department of oral pathology, oral medicine \& periodontology, Faculty of dentistry, University of \\ Malaya. \\ ${ }^{2}$ MDS, Lecturer, Oral and Maxillofacial Pathology, Mahatma Ghandhi Dental College and hospital, Jaipur.
}

Correspondence:

Department of Oral Pathology,

Mahatma Ghandhi Dental College and hospital,

RIICO, Institutional area, Sitapura,

Jaipur, Rajasthan, India - 302022

E-mail add: kundendu@hotmail.com
George T, Bishen KA. Atypical ameloblastoma - an enigma in diagnosis: review of literature and report of a case. J Clin Exp Dent. 2011;3(1):e60-5. http://www.medicinaoral.com/odo/volumenes/v3i1/jcedv3i1p60.pdf

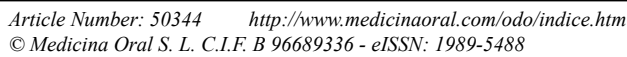

\begin{abstract}
Ameloblastoma, a benign neoplasm of jaw bones is the most common of all odontogenic tumors. Its pathology is generally well understood and is easy to diagnose. This article presents a rare case of an ameloblastoma with atypical features depicting an example of the diagnostic difficulty posed by some ameloblastomas and briefly reviews the classification and literature of odontogenic malignancies. This case is unusual in the fact that although the clinical presentation was suggestive of a malignancy, the histological features were not sufficient to warrant the lesion as malignant. Albeit, the features of epithelial dedifferentiation were evident at post operative histopathological evaluation but no proof was available to authenticate frank metastasis or carcinoma. The case was diagnosed as an atypical ameloblastoma and frequent follow up was recommended. This article discusses about ameloblastic carcinoma, other odontogenic malignancies and emphasizes the need for standardization and quantification of the fundamental concepts of odontogenic malignancies for more reliable and early diagnosis for better treatment and prognosis.
\end{abstract}

Key words: Atypical ameloblastoma, proliferative ameloblastoma, ameloblastic carcinoma. 


\section{Introduction}

Of all the odontogenic tumors, the pathology of ameloblastoma - being the most common is generally well understood. However the concept of odontogenic malignancies has been a subject of considerable discussion and controversy for many years. The term ameloblastic carcinoma is used to designate a lesion with histological evidence of malignancy in primary tumor, regardless of whether it has metastasized (1). The malignant epithelial proliferation may initially be present with a resemblance to an ameloblastoma (de novo ameloblastic carcinoma) or sometimes an ameloblastoma may show features of epithelial dedifferentiation overtime (ameloblastic carcinoma ex-ameloblastoma) (2). If the anaplastic features are not sufficient to justify an ameloblastoma as malignant, it can be called as atypical ameloblastoma (3). Ameloblastic carcinoma is a rare odontogenic malignancy that challenges the diagnostic acumen of the pathologists as the understanding of the identifying histological features of the tumor is some what vague and not standardized/quantified. We herein report a rare case of ameloblastoma which had an aggressive behavior and histologically had areas of cytological atypia which warrant a more aggressive surgical approach and follow up. The purpose of this article is to emphasize the need for standardization and quantification of the fundamental concepts of odontogenic malignancies for more reliable and early diagnosis because this has a direct bearing on the treatment plan and prognosis.

\section{Case Report}

A 58 years old male patient reported to the out patient department of Manipal college of dental sciences, Mangalore in February 2007 with the chief complaint of a non tender swelling of 2 years duration on the left side of his face. The swelling had started as a small peanut size bulge and had gradually increased to the present size. The patient gave no history of fever, malaise, associated pain or parasthesia or dysphagia. The past medical history revealed that an incisional biopsy had been performed previously which was suggestive of ameloblastoma. Family history was non contributory and social history disclosed a chronic alcoholic with a habit of chewing tobacco with betel quid for past 20 years.

Clinically, patient had a medium built and was poorly nourished, conscious and cooperative. On local examination, a diffuse swelling of size $2 \times 3 \mathrm{~cm}$ was present extraorally on the left side of face corresponding to the angle of mandible. The swelling was non tender, firm in consistency and had a smooth texture. Intraoral examination revealed an unhealed extraction socket in relation to 37 and expansion of buccal and lingual cortical plates. There was no dysphagia, pain or paresthesia.

The teeth present in the area showed no mobility or loosening. Left submandibular lymph nodes were pal- pable and tender. Radiographic examination of the mandible revealed a multilocular destructive lesion in 37,38 region involving the body and ramus of mandible and 36 showed root resorption. The chest radiographs did not demonstrate pulmonary lesions and hematological and biochemical investigations were unremarkable. Ultrasound revealed a fatty liver.

Based on the above findings, a diagnosis of ameloblastoma was made and patient underwent a hemimandibulectomy procedure and submandibular glands were also removed. The excised tissue was sent for further histopathological evaluation. Postoperative recovery was uneventful and patient was advised a follow up examination after 3 months.

The excisional tissue grossly showed a large swelling extending from 36 to the angle of mandible. The buccal side showed a large cystic cavity where as the lingual aspect showed a solid proliferation of the tumor mass showing necrotic area in centre. The occlusal aspect showed 33, 34, 35, 36 and an ulcerated area posterior to 36 (unhealed extraction socket) (Fig. 1).

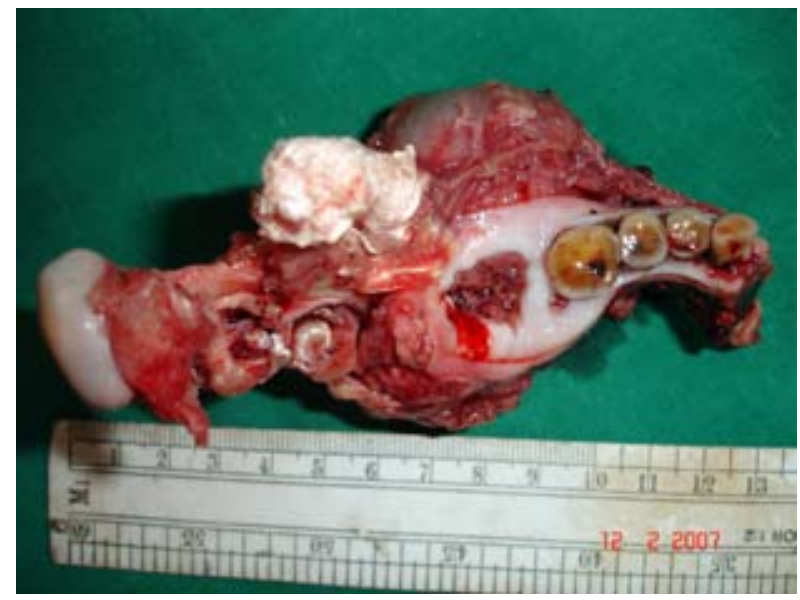

Fig. 1. Gross appearances of the specimen from occlusal view showing expansion of the buccal and lingual cortical plates with unhealed socket in relation to 37

Microscopically, the tissue sections from the buccal aspect showed a characteristic lining suggestive of a unicystic ameloblastoma consisting of a basal layer of tall columnar ameloblast like cells exhibiting reversal of polarity. The superficial layers consist of several rows of stellate reticulum like cells. Focal areas of lining epithelium showed flattened basal cells. Proliferations of the lining epithelium into the lumen as well as underlying connective tissue were also observed. The mural as well as the luminal proliferations showed plexiform ameloblastoma with areas of microcyst formation arising as a result of stromal degeneration. The stromal blood vessels showed degenerative changes appearing only as ghostly outlines surrounding few inflammatory cells.

The sections from the unhealed socket area revealed ulcerated parakeratinised stratified squamous epithelium 
with epithelial proliferation noted at the margin of ulcer. Deeper in the connective tissue the epithelial proliferation from the margin of ulcer merged with the plexiform ameloblastoma.

The sections taken from the lingual aspect revealed a follicular ameloblastoma showing numerous small and large sized ameloblastomatous follicules with a central core of stellate reticulum like cells and areas of cystic degeneration. Sheets of odontogenic epithelial cells within the connective tissue demonstrated extensive cellular pleomorphism with cells varying from ovoid to spindle in shape (Fig. 2). Occasional mitosis (typical and atypical) was evident within the sheets of odontogenic epithelium along with other features like increased nuclear cytoplasmic ratio, prominent nucleoli (Fig. 2).

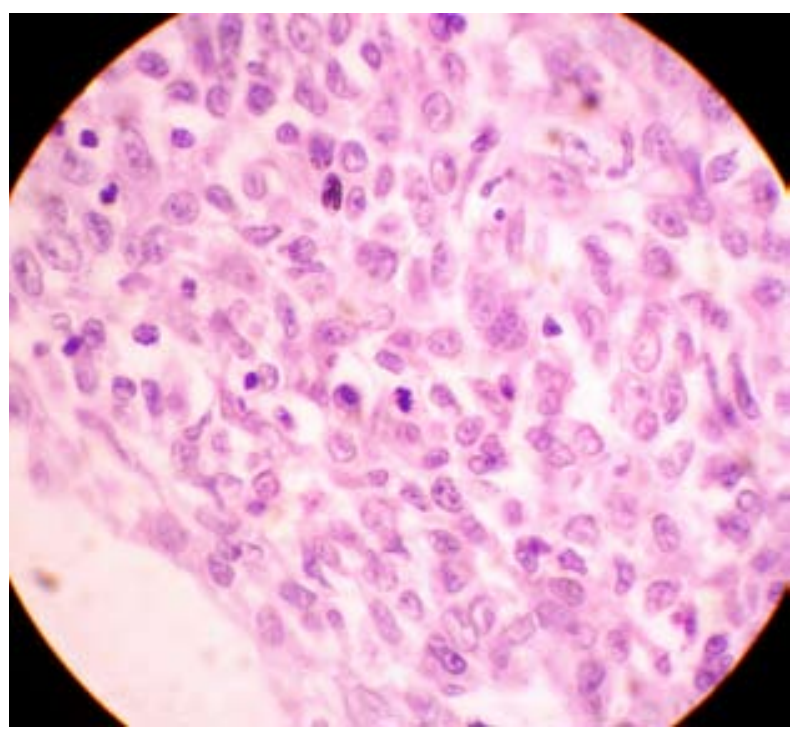

Fig. 2. Dysplastic cells exhibiting occasional atypical mitoses with cellular pleomorphism

In addition, dominant areas on lingual aspect showed sheets of squamoid cells exhibiting dyskeratosis with interspersed areas of extensive hemorrhage, degeneration and necrosis (Fig. 3). Few multinucleated giant cells were also evident. The submandibular nodes were completely free of tumor. Based on the above findings a diagnosis of atypical ameloblastoma was given.

\section{Discussion}

The typical ameloblastoma is a histologically benign tumor but it has considerable tendency to recur and even cause death by invasion of vital structures. Although it is locally invasive, it rarely shows metastatic dissemination. In a malignant ameloblastoma both the primary as well as the metastatic deposits has the typical histological features of ameloblastoma. On the other hand, histological evidences of malignancy in the primary or recurrent tumor, regardless of whether it has metastasized, characterize it as an ameloblastic carcinoma (1).

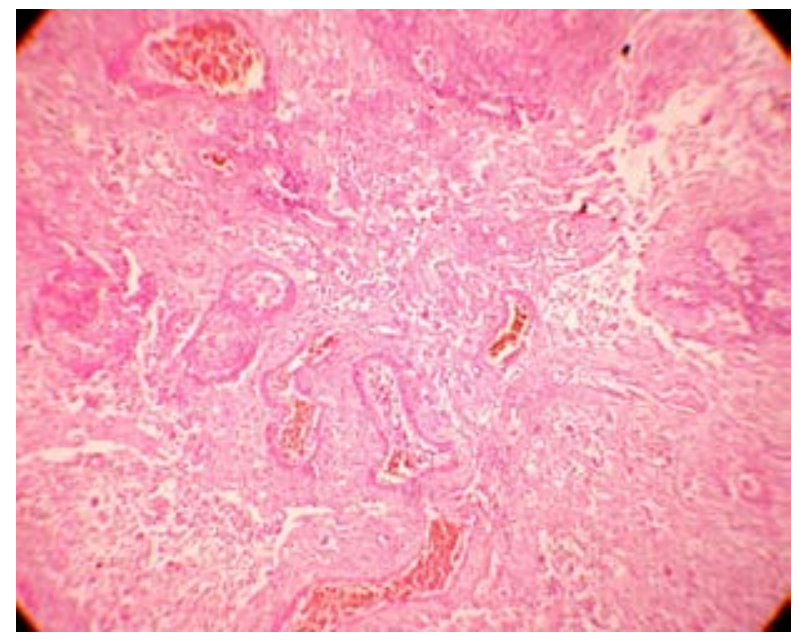

Fig. 3. Areas of degeneration, hemorrhage and necrosis

However, the epithelial dedifferentiation as seen in the present case, is not enough to warrant the ameloblastoma as malignant. Such tumors can be called as atypical ameloblastoma (3).

Ameloblastic carcinoma is a malignant odontogenic tumor, the diagnosis of which is as enigmatic, as was its position in the classification of odontogenic malignancies. The term Ameloblastic carcinoma was acknowledged by the World Health Organization (WHO) (2) as late as in 2002. The classification of odontogenic malignancies and the concept of malignancy in ameloblastoma is a controversial topic which has been discussed for long. Various classifications have been developed to categorize ameloblastic carcinomas (2-5).

The WHO published its classification for malignant neoplasms and other tumors related to the odontogenic apparatus in 1972 and recognized the following subtypes:

Odontogenic carcinoma

A. Malignant ameloblastoma

B. Primary intraosseous carcinoma (PIOC)

C. Other carcinoma arising from odontogenic epithelium including those arising from odontogenic cyst In this initial attempt to classify odontogenic carcinomas, the WHO failed to specifically delineate ameloblastic carcinomas. This WHO classification system recognized and distinguished PIOC from malignant ameloblastoma but it did not consider the possibility that a squamous cell carcinoma could arise in a pre-existing ameloblastoma and had no sub-division under which such entities could be placed.

Initially, the term ameloblastic carcinoma was introduced to describe ameloblastomas in which there had been histological malignant transformation in association with less differentiated evidence of metastatic growths. Such tumors showed features of ameloblastoma intermingled with those of carcinoma.

Elzay (4) in 1982 suggested that the WHO classification 
be modified to permit the separation and recognition of closely related entities. So he classified all intraosseous carcinoma under the heading of PIOC and then those tumors were subclassified and subtyped according to histological evidence of origin. The proposed classification was as follows:

Primary Intraosseous Carcinoma

Type 1 - Arising ex odontogenic cyst

Type 2 - Arising ex ameloblastoma

a) Well differentiated - Malignant ameloblastoma

b) Poorly differentiated - Ameloblastic carcinoma Type 3 - Arising de novo

a) Non-keratinizing

b) Keratinizing

Accordingly, the PIOC type II recognized the potential for malignification of ameloblastoma with varing degree of differentiation. Hence II A was reserved for tumors which histologically demonstrated proper ameloblastoma in the jaw and in any metastatic lesion. In essence, such tumors would be well differentiated where as the type II B sub classification was reserved for less differentiated tumors having histological features of ameloblastoma and squamous cell carcinoma concomitantaly. Two years later, in 1984, Slootweg and Müller (5) published a paper reviewing 42 cases of malignant ameloblastoma (according to WHO) and adding 2 more to the list. They pointed out that some cases of PIOC may have areas that are morphologically similar to malignant ameloblastoma. Thus there is a possibility that a PIOC would have been classified as a malignant ameloblastoma if metastasis had occurred or as a PIOC if metastasis had not occurred. They also pointed out that matters had been further complicated because of no distinction between malignant ameloblastoma and ameloblastic carcinoma. They said that the WHO classification of odontogenic carcinoma should be revised in some aspects. They advocated the modification proposed by Elzay (4) and proposed a slight modification taking in account the various possible origins of PIOC. So the classification they proposed was:

Primary Intraosseous Carcinoma

Type 1 - Primary intraosseous carcinoma ex odontogenic cyst

Type 2 - Primary intraosseous carcinoma arising ex ameloblastoma

a) Malignant ameloblastoma

b) Ameloblastic carcinoma, arising de novo, ex ameloblastoma or odontogenic cyst

Type 3 - Primary intraosseous carcinoma arising de novo

a) Non-keratinizing

b) Keratinizing

So, they accepted that malignant ameloblastoma and ameloblastic carcinoma be included under the encompassing term "PIOC ex ameloblastoma" as proposed by
Elzay (4). And they proposed that for giving a correct diagnosis, the taxonomic problem can be avoided by assuming that ameloblastic carcinomas can arise not only de novo or from a well differentiated ameloblastoma but also from other sources of odontogenic epithelium eg. odontogenic cyst. Thus, the main difference between Elzay's (4), and Slootweg and Müller's (5) schemes relates to the minor point of histogenesis.

In 1992, a modified WHO classification system for the odontogenic carcinomas was published (7), including the following categories:

Odontogenic carcinoma

A. Malignant ameloblastoma

B. Primary intraosseous carcinoma

(de novo, ex ameloblastoma, ex odontogenic cyst)

C. Malignant variants of other odontogenic epithelial tumors

D. Malignant changes in odontogenic cyst

Inspite of all the proposals made, it failed to recognize ameloblastic carcinoma as a separate entity. Since the WHO classification did not recognize the existence of ameloblastic carcinoma, all such lesions had to be classified either as PIOC or as malignant ameloblastoma. Because of this overlapping of terminologies, the potential of the histological diagnosis to distinguish the biologic behavior, prognosis and treatment plan of the ameloblastic carcinoma and closely related tumors was hindered. This taxonomic problem continued for a decade and finally ended in 2002 when ameloblastic carcinoma was recognized by the WHO in its revised version by Philipsen and Reichart (2).

The categories identified in the WHO's revised version 2002 (2) were:

Odontogenic carcinoma

A. Metastasizing ameloblastoma

B. Ameloblastic carcinoma

* Primary (=de novo)

* Carcinoma ex ameloblastoma (=dedifferen-

tiated)

* Peripheral

C. Primary intraosseous carcinoma

* Solid

* Cystogenic

D. Ghost cell odontogenic carcinoma

E. Clear cell odontogenic carcinoma

Thus, the term "ameloblastic carcinoma" was used by Shafer, Elzay(4), Slootweg and Müller's (5), primarily to convey the presence of cytologic features of malignancy in an ameloblastoma came into being and got recognized.

Ameloblastic carcinoma is considered to be a rare odontogenic malignancy. Akrish et al. (6) analyzed all the published cases in English language literature between the years 1984 and 2004 to find only 37 cases reported in addition to the case presented in the paper (making a 
total of 38 cases). It might be that, because of the prolonged controversy around ameloblastic carcinoma it was either wrongly categorized or less reported. Unfortunately, as a consequence of this, the understanding of the identifying clinical and histological features of the tumor is still some what vague and not standardized/ quantified.

Although Corio et al. (1) had reported the mean age of occurrence to be 30.1 years with no gender predilection, recently Akrish et al. (6) reported the mean age of occurrence to be 52 years and the male to female ratio being 1.5 to 1 . The posterior mandible was the most favored site in both the reports. Radiographically the tumor resembles ameloblastoma and mostly appears as a multilocular radiolucency. Clinically the patients mostly complain of expansion, a hard mass, rapid growth, facial asymmetry, a nonhealing extraction site, an ulcer, a fistula or perforation of the cortex. These clinical features though suggestive of a malignancy, are again not pathognomonic. In the present case also the patient had most of these features.

Histologically ameloblastic carcinoma demonstrates more cytologic atypia and mitotic activity than ameloblastoma. It includes the features of epithelial dedifferentiation. There is lack of evidence of reverse polarization, sheets of disordered mitotically active small basaloid cells with dark nuclei; larger squamoid or polygonal cells with vesicular nuclei; or elongated spindled epithelial cells (6). The histological diagnosis is not an easy one and a pathologist must rule out a lexicon of differential diagnosis which includes the typical ameloblastoma, metastatic carcinoma to the jaw, intra bony extension of a surface mucosal carcinoma, central salivary gland tumor, PIOC, acanthomatous ameloblastoma, keratoameloblastoma, squamous odontogenic tumor, and calcifying epithelial odontogenic tumor. In the present case also all these differential diagnoses were ruled out as elaborated by Coiro et al. (1)

Until 2004, the terms aggressive or proliferative ameloblastoma, ameloblastic carcinoma and atypical ameloblastoma were used synonymously by some investigators (7). In 2004 Slater (3) suggested that ameloblastomas which exhibit basilar hyperplasia and an increased mitotic index should be designated as "atypical ameloblastomas" or "proliferative ameloblastomas" because these findings are probably insufficient to permit a diagnosis of ameloblastic carcinoma in the absence of nuclear pleomorphism, perineural invasion or other histologic evidence of malignancies (3). The picture is further complicated by the fact that although the presence of abundant mitotic figures is one of the most important diagnostic criteria, the prognostic significance of this is not known (8).

The enigma about the diagnosis of ameloblastic carcinoma is further aggravated because the diagnostic criteria to distinguish between ameloblastoma, atypical ameloblastoma and ameloblastic carcinoma are not standardized/ quantified. Many important questions/concepts remain unanswered e.g. Is the diagnosis of atypical ameloblastoma of any relevance for treatment options? What features are necessary to differentiate between these closely related lesions? Which histologic feature is a sine qua non for diagnosing a particular odontogenic malignancy? If two lesions have an overlapping features e.g. Presence of mitotic figures or a high proliferative index, how many mitoses per high power field or what percent of cells showing high index should distinguish two closely related lesions? We hereby strongly propose that the fundamental concepts of odontogenic malignancies should be standardized and quantified for more reliable and early diagnosis because this has a direct bearing on the treatment plan and prognosis.

To conclude, the present case is an example of the diagnostic difficulty posed by some ameloblastomas. This case is unusual in the fact that although the age, rapidity of growth and clinical presentation were suggestive of a malignancy, the histological features were not sufficient to warrant the lesion as malignant. Albeit, the features of epithelial dedifferentiation were evident at post operative histopathological evaluation but no proof was available to authenticate frank metastasis or carcinoma. Hence in the light of current concepts the tumor was categorized as an ameloblastoma with atypical features and frequent follow up was recommended. 


\section{References}

1. Corio RL, Goldblatt LI, Edwards PA, Hartman KS. Ameloblastic Carcinoma: A clinicopathologic study and assessment of eight cases. Oral Surg Oral Med Oral Pathol. 1987;64:570-6.

2. Philipsen HP, Reichart PA. Revision of the 1992-edition of the WHO histological typing of odontogenic tumours. A suggestion. J Oral Pathol Med. 2002;31:253-8.

3. Slater LJ. Odontogenic malignancies. Oral Maxillofac Surg Clin North Am. 2004; 16:409-24.

4. Elzay RP. Primary intraosseous carcinoma of the jaws. Review and update of odontogenic carcinomas. Oral Surg Oral Med Oral Pathol 1982; 54:299-303.

5. Slootweg PJ, Müller H. Malignant ameloblastoma or ameloblastic carcinoma. Oral Surg Oral Med Oral Pathol. 1984;57:168-76.

6. Akrish S, Buchner A, Shoshani Y, Vered M, Dayan D. Ameloblastic carcinoma: report of a new case, literature review, and comparison to ameloblastoma. J Oral Maxillofac Surg. 2007;65:777-83.

7. Ameerally P, McGurk M, Shaheen O. Atypical ameloblastoma: report of 3 cases and a review of the literature. Br J Oral Maxillofac Surg. 1996;34:235-9.

8. Gardner DG.Some current concepts on the pathology of ameloblastomas. Oral Surg Oral Med Oral Pathol Oral Radiol Endod. 1996;82:660-9. 